\title{
Cattle Sperm Classification Using Transfer Learning Models
}

\author{
Jessica S. Velasco ${ }^{1}$, Nilo M. Arago ${ }^{2}$, Roan M. Mamba ${ }^{3}$, Maria Victoria C. Padilla ${ }^{4}$, \\ John Peter M. Ramos ${ }^{5}$, Glenn C. Virrey ${ }^{6}$ \\ 1,2,3,4,5,6 Department of Electronics Engineering, Technological University of the Philippines, Manila 1000, \\ Philippines \\ 1 jessica_velasco@tup.edu.ph, ${ }^{2}$ nilo_arago@tup.edu.ph, ${ }^{3}$ roan_mamba@tup.edu.ph, \\ ${ }^{4}$ mariavictoria_padilla@tup.edu.ph, ${ }^{5}$ johnpeter_ramos@tup.edu.ph, ${ }^{6}$ glenncalvin_virrey@tup.edu.ph
}

\begin{abstract}
This paper focused on classifying sperm and white blood cells (WBC) through image processing by utilizing different architectures of Transfer Learning Model (TLM). For image classification the researchers used microscopic images of sperm. A total of 602 image datasets were used for training and testing in deep learning with different convolutional network models. The models used are: InceptionResNetV2, Xception, DenseNet121, DenseNet169, MobileNetV1, InceptionV3, and DenseNet201. The classification of sperm and WBC is implemented successfully. The following is observed in the evaluation of these models: confusion matrix, loading time, weight size, and accuracy. From these evaluations: the highest model to consider for true positive is InceptionResnetV2. The accuracy of $98.3 \%$ is obtained by this model. However, the DenseNet121 also has comparable results with an accuracy of $95 \%$ considering its weight size of $93.49 \mathrm{MB}$ as compared to InceptionResnetV2 of 641.93 MB.
\end{abstract}

Key words: Convolutional Neural Network, Deep Learning, Transfer Learning, Cattle Sperm Classification; Morphology, White Blood Cells

\section{INTRODUCTION}

One of the world's most vital agricultural enterprises is the cattle industry. This includes cattle production for various purposes, such as meat, dairy, hides, and other products. A professor at Cornell University, William Lesser stated that cattle had been raised for a thousand years and had given four important roles to the early community. Such are source of high-grade protein, the means to store food that can be consumed indirectly by humans, skins for clothing and shoes and motive power (traction) [1].

As of January $1^{\text {st }}, 2019$, as stated in the report of the Philippine Statistics Authority on cattle situation, the inventory of cows was estimated at 1.10 million heads. In Fig. 1, total cattle stock was 43.4 percent, and a 56.6 percent remaining affected by yearlings, bulls, heifer, and others (bulls to be castrated and ready for breeding) [2]

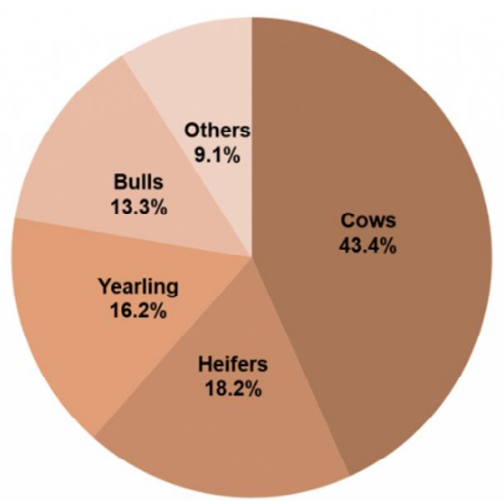

Figure 1: Distribution of Cattle Inventory as of January 2019

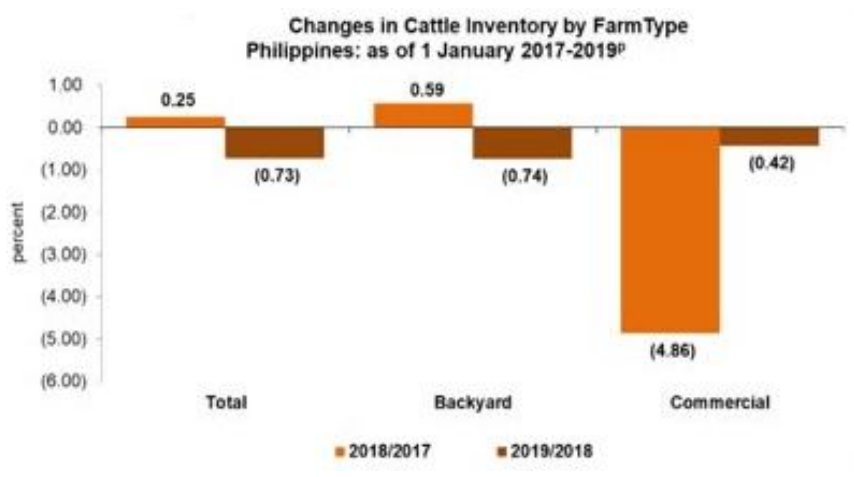

Figure 2: Farm Type Cattle Inventory

Figure 2 shows the changes in cattle inventory from 20172019. 2.54 million heads was the total number of cattle stock as compared to the figures of 2.55 million heads from prior year, there's a decline of 0.73 percent [2]. Hence, infertility of a cattle really is one of the major hindrances in livestock production for it directly affects dairy farm profitability and the improvement of national economy, as well as the lifestyle in the rural and urban communities. 
Cattle infertility is characterized as the ability to produce viable offspring reduced or absent. Infections which decrease ovulation levels, fertilization levels, embryonic survival rates, fetal or perinatal survival rates cause infertility in cattle. [3]. The procedure for herd infertility examination consists evaluation of breeding history, feeding and management practices, and examination of health records, herd, and laboratory results to make an objective diagnosis [4]. In addition, due to cryopreservation, low fertility is widely accepted [5]. The reduction occurs in a proportion of the remaining subpopulation from both lower viability post-thaw and sublethal dysfunction. There are varied reasons for fertility loss.

Computer-assisted semen analysis produce values for sperm parameters faster and more accurately than those obtained with conventional methods of semen analysis. In modern years, science progressed rapidly mainly in computer and video processing; therefore, the functionality and accuracy of CASA system's latest versions are dramatically enhanced and provide more knowledge on the different sperm movement characteristics. Due to the sperm motility 's key role in the reproductive cycle, theses systems have capability to allow us to enter a new diagnostic andrological age and predict the semen's ability to fertilize [6].

There are several literatures relating to feature extraction used in sperm classification using the artificial neural network. An evaluation of fertility data where the penetrak and zona-free hamster egg penetration assay were used [7]. A seminal quality prediction in which the semen samples were classified to optimize the structure of artificial neural network using a genetic algorithm [8]. Fertility quality prediction based on Sperm Whale Optimization Algorithm (SWA) [9]. A novel deep learning algorithm for detecting sperm morphology using images of human sperm cells [10].

Cattle semen analysis is performed as part of breeding. The proponents, together with the help of San Juan, Batangas Municipal Agricultural Office Farmer's Information and Technology Services (FITS) Center in data gathering, will perform deep learning using transfer learning models to visually infer whether it is normal, abnormal or WBC by getting images obtained manually from the microscope and classify each image to its right category.

\section{RELATED WORKS}

\subsection{Deep Learning}

A well-known machine learning technique and has been mostly used in many applications to solve various complex problems requiring high sensitivity and accuracy, especially in the medical field which has paid a great deal of attention to researchers in recent years. In the paper where brain tumor was classified through deep learning, Among the commonly used deep learning architectures is the Convolutional Neural Network (CNN) for the classification of brain tumors into three classes (Meningioma, Pituitary, and Glioma Tumor) of a dataset of $3064 \mathrm{~T} 1$ weighted contrast-enhanced brain MR images [11].

In the paper "Study of Detection of Various types of Cancers by using Deep Learning: A Survey", the proponents concluded that the only way to reduce the death ratio of cancer, is by early detection of it. Deep learning methods were used by the author of 22 papers which helps to detect the cancer early [12].

\subsection{Transfer Learning}

In spite of the enormous resources needed to train models of deep models, transfer learning is a widely used deep learning. Transferring of information from a similar task which has already been taught to develop a new task [7].

In the study "Canine Semen Evaluation using Transfer Learning Models" had used different kinds of transfer learning models (TLM) for canine semen evaluation and integrating the performance of Deep Learning algorithms. A 332 total of datasets were used for training and testing of different convolutional network models [13].

\subsection{Keras Platform}

In the study "Fully convolutional networks for segmenting images from an embedded camera" [14], Python, Keras, and Theano was used in implementing and prototyping Fully Convolutional Networks. This paper describes an FCN to separate images of a compact stereo imaging sensor mounted on a robot to provide functions for low-level computer vision.

The Keras Library was used to model the design of the network, speeding up the process of finding a network with good precision and low computational resource consumption. To retrain the flower category datasets, transfer learning method was used which can significantly increase the accuracy of flower classification.

\subsection{Xception}

Xception is an Inception-inspired deep convolutional neural network. This far exceeds InceptionV3 on the ImageNet dataset and on a broader dataset of image classification of 350 million images and 17,000 classes [15].

\subsection{DenseNet121}

In the research "Classification of TrashNet Dataset Based on Deep Learning Models" [16], deep learning models were used to classify recyclable garbage and the best results were seen in the DenseNet121 with a test accuracy of $95 \%$ using fine-tuning.

\subsection{InceptionResNetV2}

This is the ensemble of three residual and one Inception V4.It is developed by Alex Alemi, Christian Azegedy, Sergey Ioffe, and Vincent Vanhoucke [17]. In the study "PolyNet: A 
Pursuit of Structural Diversity in Very Deep Networks" [18], the researchers proposed a new module family, namely the PolyInception.

\subsection{DenseNet169}

Huang et al introduced the DenseNet169 architecture [19]. In the study "Pneumonia Detection Using CNN based Feature Extraction" [20], The DenseNet169 model has the ability to view function maps from all previous layers, thereby delivering the best results.

\subsection{MobileNetV1}

MobileNetV1 has depth convolution wherein a single filter applies to every data channel. It also has a standard convolution, which in one step combines both inputs and filters into a new set of outputs [21].

In the study "A Smartphone-Based Skin Disease Classification Using MobileNet CNN" [22], a total of 3,406 images were collected by the proponents and it is considered as imbalanced dataset due of the unequal number of images on its classes. To further improve the MobileNet's accuracy, different sampling methods and pre-processing of input data were explored.

\subsection{InceptionV3}

In the research "Inception-v3 for flower classification" [23], to classify flowers the proponents used TensorFlow platform Inception-v3 model. The model's classification accuracy on Oxford-I7 flower dataset is $95 \%$, and $94 \%$ accuracy on Oxford102 flower dataset, which is greater than any other method.

\subsection{DenseNet201}

Two separate deep learning approaches are tested on the dataset of breast cancer in the study "Comparison of two different deep learning architectures on breast cancer" [24]. For training there are 20748 images and for evaluation there are 5913 images and based on the results obtained, DenseNet-201 architecture has a $92.24 \%$ accuracy.

\section{METHODOLOGY}

\subsection{Microscopic Analysis}

Microscopic analysis is performed since it is very important in semen analysis. It measures the quality and quantity of the moving cells called sperm. Therefore, the clinical condition determines the extent of analysis for accurate results.

Figure 3 shows the preparation of semen analysis. The system's main input is the semen sample which will be put on a slide. To keep semen constituents from disintegrating, the semen to use must be as fresh as possible. Consistency of the sediment volume put on the slide must be observed correctly. For conventional method of glass-slide, $6 \mu \mathrm{L}$ $(0.006 \mathrm{~mL})$ is the prescribed volume protected by an $18 \times 18$ mm glass cover slip. If $10 \mu \mathrm{L}$ volume of semen is to be use then a $22 \times 22$ cover slip is required [25]. The semen sample will undergo microscopic analysis such as sperm concentration, morphology, and motility.

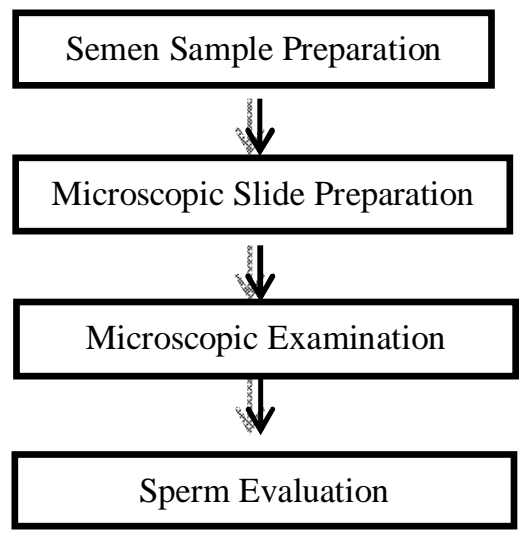

Figure 3: Steps in preparation for Semen Analysis

\subsection{Microscopic Images}

The images used in this study are microscope-collected images. Figure 4 shows a sample of sperm images classified as normal and abnormal, and the WBC.
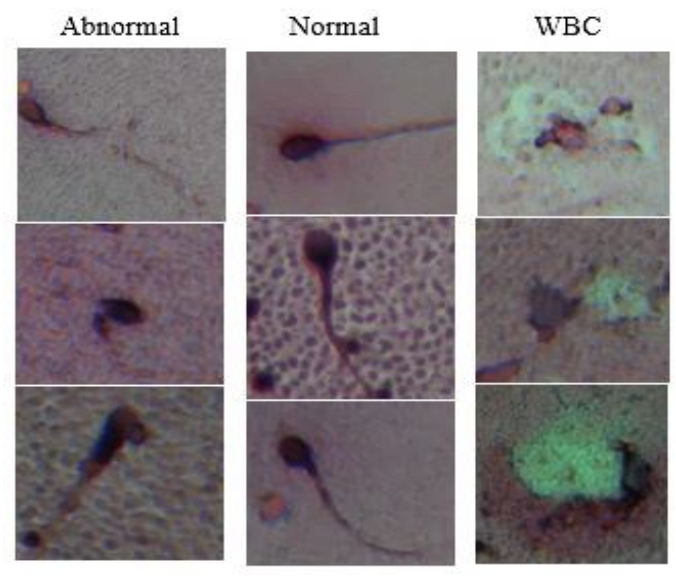

Figure 4: Sample images of dataset: Abnormal, Normal, and WBC

\subsection{System Model}

This study's system model will start in the base model by loading the datasets. The researchers used the following models: Xception, DenseNet121, InceptionResNetV2, DenseNet169, MobileNetV1, InceptionV3, and DenseNet201. The datasets have three categories: normal sperm, abnormal sperm, and WBC. The network is now trained and evaluated for datasets classification according to their category. The classifier will be loaded into the system upon testing and is used for classification by category. Evaluation for each category shall take place according to the convolutional model. 
Jessica S. Velasco et al., International Journal of Emerging Trends in Engineering Research, 8(8), August 2020, 4325 - 4331

\subsection{Data Collection}

The microscope with $4 \mathrm{x}$ and $10 \mathrm{x}$ magnifier was used to collect the microscopic images of the semen samples. A total of 602 images were obtained. The breakdown of semen sample images is shown in Table 1.

Table 1: Dataset

\begin{tabular}{|c|c|c|c|c|}
\hline $\begin{array}{c}\text { Semen } \\
\text { Sample }\end{array}$ & $\begin{array}{c}\text { Number } \\
\text { of } \\
\text { Images }\end{array}$ & $\begin{array}{c}\text { Train } \\
\text { Data }\end{array}$ & $\begin{array}{c}\text { Test } \\
\text { Data }\end{array}$ & $\begin{array}{c}\text { Validation } \\
\text { Data }\end{array}$ \\
\hline $\begin{array}{c}\text { Normal } \\
\text { Sperm }\end{array}$ & 234 & 187 & 47 & 46 \\
\hline $\begin{array}{c}\text { Abnormal } \\
\text { Sperm }\end{array}$ & 214 & 171 & 43 & 45 \\
\hline $\begin{array}{c}\text { White Blood } \\
\text { Cells }\end{array}$ & 154 & 123 & 31 & 31 \\
\hline Total & 602 & 481 & 121 & 120 \\
\hline
\end{tabular}

The dataset is split into $80 \%$ for training and $20 \%$ for testing. The validation data is collected from either the training data or testing data also.

\subsection{Experiment: Feature Extraction, Training and Testing}

Keras platform will be used with Tensorflow backend in coding system. The proponents limit the epoch of 80 .

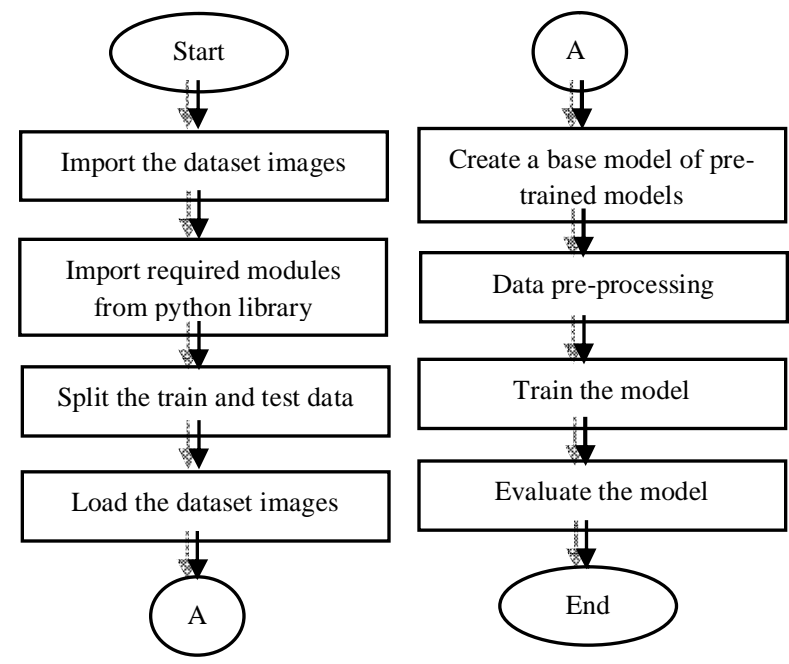

Figure 5: Program flowchart of the python code

Figure 5 shows the program starts at organizing imports such as numpy, keras, sklearn, and matplotlib. Then, Split the training and testing data by configuring the dataset into specific directories. Third, is to load sperm images from subfolders of category. Next, create a base model of different pre-trained CNN. Then, acquire the features by preprocessing the data. Next, is to configure the training and testing of the model. Lastly, after training, different architectures will be evaluated and compared based on model accuracy, comparison of losses, confusion matrix, loading time, and weight size to verify what is the best model for the sperm morphology and WBC classification.

For every model, the input images are different. A rectangular prism is the normal shape of a convolutional model. It is the product of height, width, and depth. On the otherhand, input image is equal to the product of image size and channel number. The colored images channel number is 3 while black and white channel number is 1 . Table 2 shows each model and the sizes of each input.

Table 2: Input for each Model

\begin{tabular}{|c|c|}
\hline Model & Input Image \\
\hline Xception & $224 \times 224 \times 3$ \\
\hline DenseNet121 & $224 \times 224 \times 3$ \\
\hline InceptionResNetV2 & $224 \times 224 \times 3$ \\
\hline DenseNet169 & $224 \times 224 \times 3$ \\
\hline MobileNetV1 & $224 \times 224 \times 3$ \\
\hline InceptionV3 & $299 \times 299 \times 3$ \\
\hline DenseNet201 & $224 \times 224 \times 3$ \\
\hline
\end{tabular}

\section{RESULTS AND DISCUSSION}

To compare and verify the effectives of each pre-trained convolutional neural networks, following criteria was observed: Confusion matrix, loading time, accuracy, and weight size.

\subsection{Confusion Matrices}

The confusion matrix is also called matching matrix, where a row means a projected class and the column means the real class [26]. This demonstrates the misclassification similarity between the different convolutional neural networks. The Fig. 6 shows the confusion matrices of different models over the 3 classes for sperm morphology and WBC.

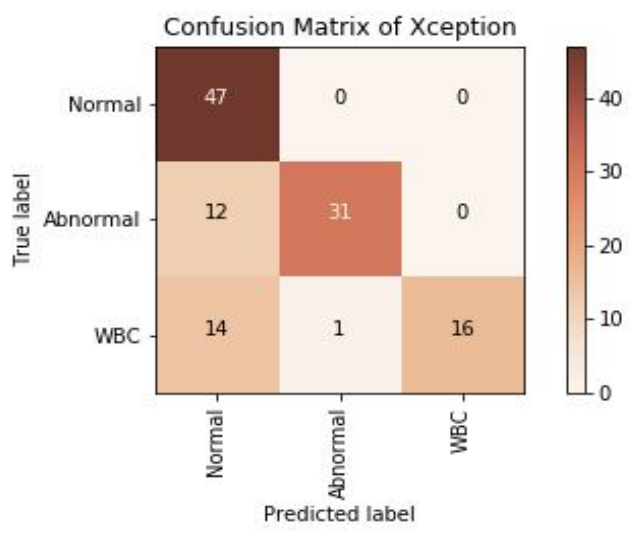

(a) Xception 
Jessica S. Velasco et al., International Journal of Emerging Trends in Engineering Research, 8(8), August 2020, 4325 - 4331

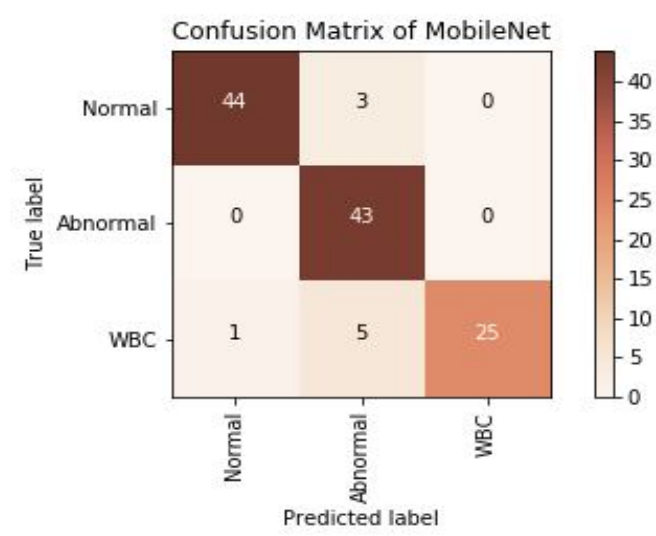

(b) MobileNet

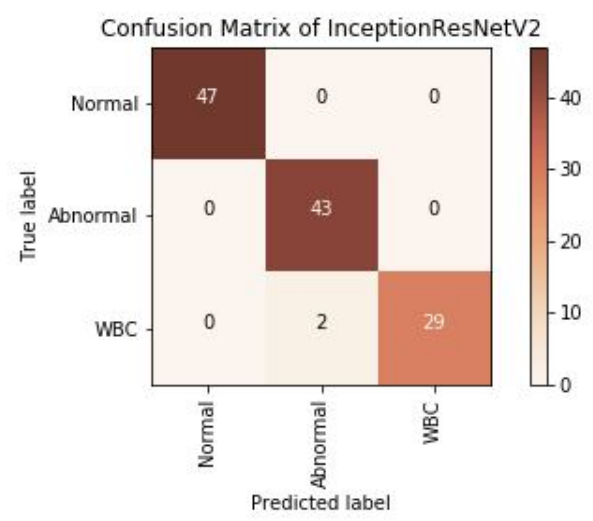

(c) InceptionResNetV2

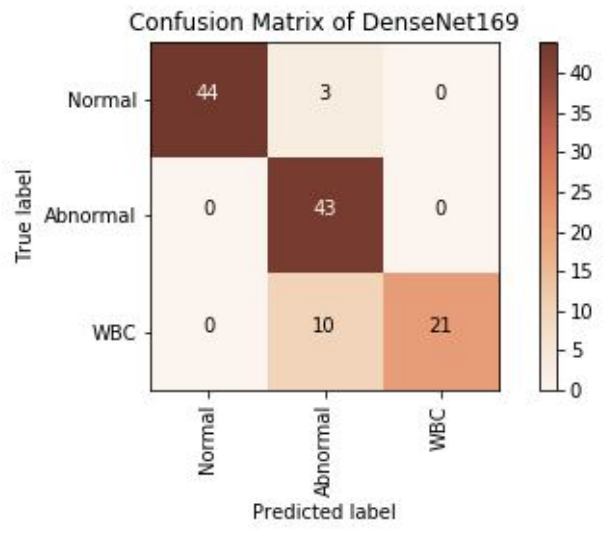

(d) DenseNet169

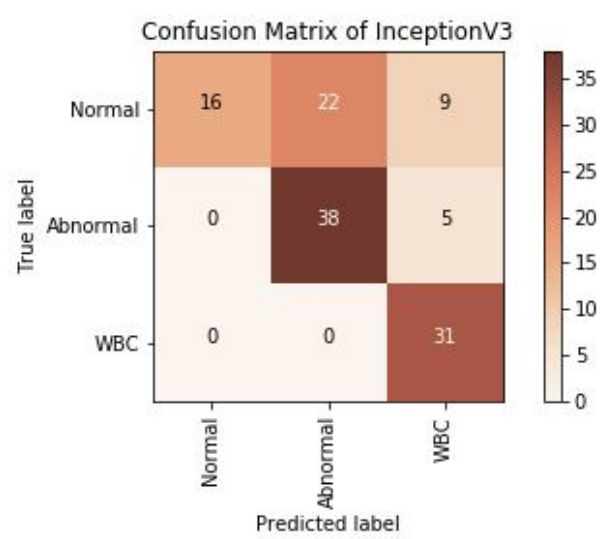

(e) InceptionV3

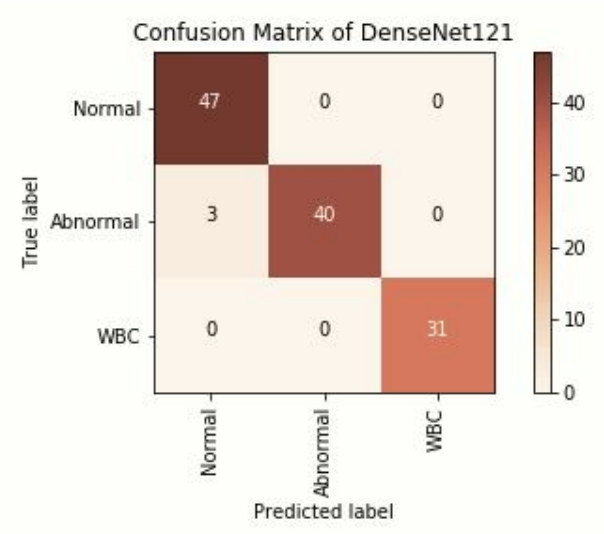

(f) DenseNet121

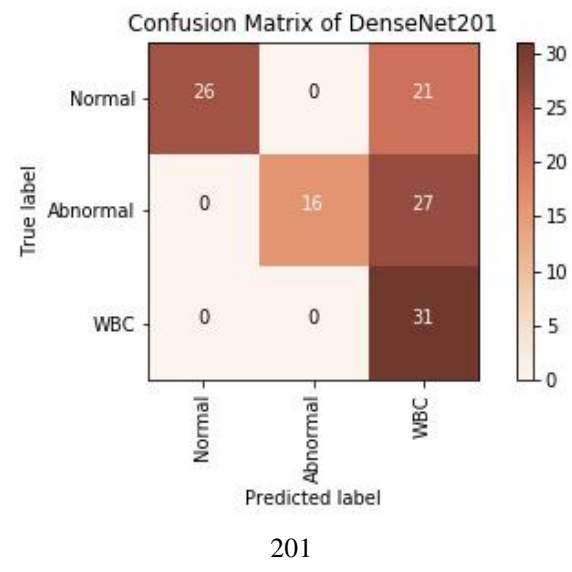

Figure 6: Confusion matrix of the sperm and WBC classification of different convolutional network models: (a) InceptionResNetV2, (b) DenseNet121, (c) MobileNetV1, (d) DenseNet169, (e) Xception, (f) InceptiontV3 and (g) DenseNet201 
Jessica S. Velasco et al., International Journal of Emerging Trends in Engineering Research, 8(8), August 2020, 4325 - 4331

\subsection{Weight Size, Loading Time, and Accuracy}

In the Table 3 , the highest performing model is the InceptionResNetV2 with an accuracy of $98.3 \%$.

Table 3: Data Analysis

\begin{tabular}{|c|c|c|c|}
\hline Model & $\begin{array}{c}\text { Weight } \\
\text { Size } \\
\text { (MB) }\end{array}$ & $\begin{array}{c}\text { Loading } \\
\text { time } \\
\text { (seconds) }\end{array}$ & $\begin{array}{c}\text { Accuracy } \\
\text { (percent) }\end{array}$ \\
\hline InceptionResNetV2 & 641.94 & 10.961 & 98.3 \\
\hline DenseNet121 & 93.49 & 7.372 & 95.0 \\
\hline MobileNet & 26.79 & 1.389 & 92.6 \\
\hline DenseNet169 & 165.16 & 10.753 & 89.3 \\
\hline Xception & 262.96 & 2.513 & 77.7 \\
\hline InceptionV3 & 274.36 & 4.748 & 70.2 \\
\hline DenseNet201 & 233 & 12.876 & 60.3 \\
\hline
\end{tabular}

While DenseNet121 and MobileNet have an accuracy of 95\% and $92.6 \%$ respectively, it is still analogous with the execution of InceptionResNetV2. These models are also considered to have the least weight size as compared to other models.

\section{CONCLUSION}

This study used transfer learning in sperm morphology and WBC classification. Pre-trained models were used such as Xception, DenseNet121, InceptionResNetV2, DenseNet169, MobileNetV1, InceptionV3, and DenseNet201. The sperm images used were taken manually which include: Normal, Abnormal and WBC. The result shows that InceptionResNetV2 has the highest accuracy of 98.3\%; however, the very lightweight models are DenseNet121 and MobileNet have an analogous accuracy of $95 \%$ and $92.6 \%$ with a very fast loading time of 7.372 and 1.389 seconds, respectively.

\section{ACKNOWLEDGEMENT}

The authors would like to thank the Technological University of the Philippines for the grant.

\section{REFERENCES}

1. W. Lesser, 1993. [Online]. Available: https://www.encyclopedia.com/plants-andanimals/agriculture-and-horticulture/agriculturegeneral/cattle-industry.

2. R. P. Bautista, "Cattle Situation Report, January-March 2019" Philippine Statistics Authority, 21 May 2019. [Online]. Available: http://www.psa.gov.ph/content/cattlesituation-report-january-march-2019-0.

3. Givens and M. Daniel, "A clinical, evidence-based approach to infectious causes of infertility in beef cattle," 2006.

4. A.Morrow and David, "Diagnosis and Prevention of Infertility in Cattle," 1970.
5. P. Watson, "The causes of reduced fertility with cryopreserved semen," 2000.

6. S. Cseh, T. Polichronopoulos and L. Solti, "PREDICTION OF BULL FERTILITY BY COMPUTER ASSISTED SEMEN ANALYSIS," 2003.

7. C. S. Niederberger, L. I. Lipshultz and D. J. Lamb, A neural network to analyze fertility data, vol. 60 , no. 2 , 1993.

8. A. A. Bidgoli, H. E. Komleh and S. J. Mousavirad, Seminal quality prediction using optimized artificial neural network with genetic algorithm, 2015.

9. E. EL-SHAFEIY, A. EL-DESOUKY and S. ElGHAMRAWY, An Optimized Artificial Neural Network Approach Based, 2018.

10. S. Javadi and S. A. Mirroshandel, A novel deep learning method for automatic assessment of human sperm images, vol. 109, 2019.

11. A. M. Alqudah, H. Alquraan, I. A. Qasmieh, A. Alqudah and W. Al-Sharu, "International Journal of Advanced Trends in Computer Science and Engineering," Brain Tumor Classification Using Deep Learning Technique A Comparison, vol. 8, 2018.

12. N. Malik, V. Bharat, S. Tiwari and J. Singla, "International Journal of Advanced Trends in Computer Science and Engineering," Study of Detection of Various types of Cancers by using Deep Learning: A Survey, vol. 8, no. 4, 2019.

13. J. S. Velasco, M. V. C. Padilla, N. M. Arago, E. P. E. D. Vera, F. E. M. Domingo and R. E. R. Ramos, "Canine Semen Evaluation using Transfer Learning Models," International Journal of Emerging Trends in Engineering Research, vol. 8, no. 7, 2020.

14. C. A. D. S. P. Rodrigues, C. Vinhal and G. d. Cruz, "Fully convolutional networks for segmenting images from an embedded camera," 2017.

https://doi.org/10.30534/ijeter/2020/173872020

15. Chollet and Francois, "Xception: Deep Learning With Depthwise Separable Convolutions," 2017.

16. R. A. Aral, Ş. R. Keskin, M. Kaya and M. Haciömeroğlu, "Classification of TrashNet Dataset Based on Deep Learning Models," 2018.

17. T. Akiba, S. Suzuki and K. Fukuda, "Extremely Large Minibatch SGD: Training ResNet-50 on ImageNet in 15 Minutes," 2017.

18. X. Zhang, Z. Li, C. C. Loy and D. Lin, "PolyNet: A Pursuit of Structural Diversity in Very Deep Networks," 2017.

19. G. Huang, Z. Liu, L. v. d. Maaten and K. Q. Weinberger, "Densely Connected Convolutional Networks," 2017.

20. D. Varshni, K. Thakral, L. Agarwal, R. Nijhawan and A. Mittal, "Pneumonia Detection Using CNN based Feature Extraction," 2019.

21. H.-C. Shin, H. R. Roth, M. Gao, Z. X. Le Lu, I. Nogues, J. Yao, D. Mollura and R. M. Summers, "Deep Convolutional Neural Networks for Computer-Aided Detection: CNN 


\section{Architectures, Dataset Characteristics and Transfer} Learning," 2016.

22. J. Velasco, C. Pascion, J. W. Alberio, J. Apuang, J. S. Cruz, M. A. Gomez, B. J. Molina, L. Tuala, A. Thio-ac and R. J. Jorda, "A Smartphone-Based Skin Disease Classification Using MobileNet CNN," International Journal of Advanced Trends in Computer Science and Engineering, vol. 8, no. 5, 2019 ..

https://doi.org/10.30534/ijatcse/2019/116852019

23. X. Xia, C. Xu and B. Nan, "Inception-v3 for flower classification," 2017.

24. F. Yilmaz, O. Kose and A. Demir, "Comparison of two different deep learning architectures on breast cancer," 2019.

25. "Semen analysis: an overview," in Basic Semen Analysis, 2002, pp. 1-4.

26. "Confusion Matrix," Wikipedia, [Online]. Available: https://en.wikipedia.org/wiki/Confusion_matrix. 